\title{
Determination of Material Properties like Permittivity and Density with Microwaves
}

\author{
Christoph Sklarczyk \\ Fraunhofer-Institute for Nondestructive Testing (IZFP), Saarbrücken, Germany \\ Email: christoph.sklarczyk@izfp.fraunhofer.de
}

Received 27 February 2014; revised 24 March 2014; accepted 14 April 2014

Copyright (C) 2014 by author and Scientific Research Publishing Inc.

This work is licensed under the Creative Commons Attribution International License (CC BY). http://creativecommons.org/licenses/by/4.0/

(c) (1) Open Access

\begin{abstract}
With the help of electromagnetic waves in deci-, centi- and millimeter-wave range (microwaves) it is possible to determine the properties of non-metallic objects like permittivity or density in a nondestructive and if necessary in a contactless way. Depending on the type of the test object the measurement can be carried out both with low-cost narrowband or more expensive wideband devices and sensors. To get the characteristic value in most cases it is necessary to calibrate the test device with the help of reference materials. It is recommendable to sustain a constant distance (lift-off or standoff) between the antenna of the sensor and the test object. The paper deals with the characterization of asphalt, especially the determination of its density.
\end{abstract}

\section{Keywords}

Nondestructive, Microwaves, Permittivity, Density, Sensor

\section{Introduction}

Microwaves can be defined as electromagnetic waves in the frequency range between $0.3 \mathrm{GHz}$ and $300 \mathrm{GHz}$. The physical principles of their interaction with gases, liquids and solids are well known since a long time [1] [2]. Phenomena like scattering, reflection, transmission, refraction or polarization strongly depend on the electromagnetic properties of the material (permittivity, permeability, electric conductivity) and the geometry of the test object. Normally the electromagnetic material constants are strongly correlated to chemical, physical and mechanical material properties like chemical composition, material density, porosity, cure state, moisture content etc. [3]-[5]. Therefore, with the help of microwaves many properties of the test object can be retrieved in a non-destructive and, if needed, a contact-free way. In microwave domain usually only dielectrics and materials with low electric conductivity can be investigated. The interaction between the microwaves and the test object can take place in free space, i.e. in far field, where the waves are approximately plane, or in near field of the an- 
tenna with more spherical waves.

In contrast to normal ultrasonic testing (UT) based on piezoelectric effect no coupling medium is needed between the microwave antenna and the test object. This distance can be much larger than that in UT. Usually the used radiation power is very low (mW range) and thus harmless to humans.

\section{Microwave Systems}

Broadband microwave methods (radar) allow for setting temporal filters and thus for eliminating disturbance signals (clutter). However, there are many applications where no disturbing scatterers, e.g. the reinforcements in concrete structures, are to be expected. In these cases low-cost fixed frequency microwave sensors or radar sensors with a very limited bandwidth can be used. The paper describes the characterization of asphalt layers with microwaves with both an ultrawideband (UWB) device and with narrowband fixed frequency sensors.

The used UWB system was a ground penetrating radar (GPR) system from Malå (type Pro-Ex) which was equipped with a ground-coupled $2.3 \mathrm{GHz}$ antenna for both transmission and reception.

Fixed-frequency sensors are more simple constructed and less expensive than the broad-band systems. Their frequency often falls into the royalty-free frequency bands like $2.45 \mathrm{GHz}$ or $5.8 \mathrm{GHz}$ (ISM-bands). Figure 1 gives an example of fixed frequency sensors which were used in monostatic mode (only one antenna for transmission and reception).

\section{Materials}

To ensure the quality of asphalt roads the density of the uppermost layer should be known. As example of use in the domain of quality assurance asphalt specimens with different densities have been chosen. The specimens were made from two asphalt types (SMA 11 S, AC 16 BS) and were positioned on wooden chip boards. The dimensions of the completely dry specimens were: length $31 \mathrm{~cm}$, width: $26 \mathrm{~cm}$, thickness: $4 \mathrm{~cm}$ (SMA $11 \mathrm{~S})$ and $6 \mathrm{~cm}$ (AC $16 \mathrm{BS}$ ). The experiments were run in the antenna near field whose length is approximately given by $\mathrm{r}$ $<2 \mathrm{~d}^{2} / \lambda$ (d: largest dimension of antenna, $\lambda$ : wave length).

\section{Experimental Results and Discussion}

\subsection{Broadband Measurements}

The measuring results with the GPR show an approximately linear relationship between the signal amplitude in

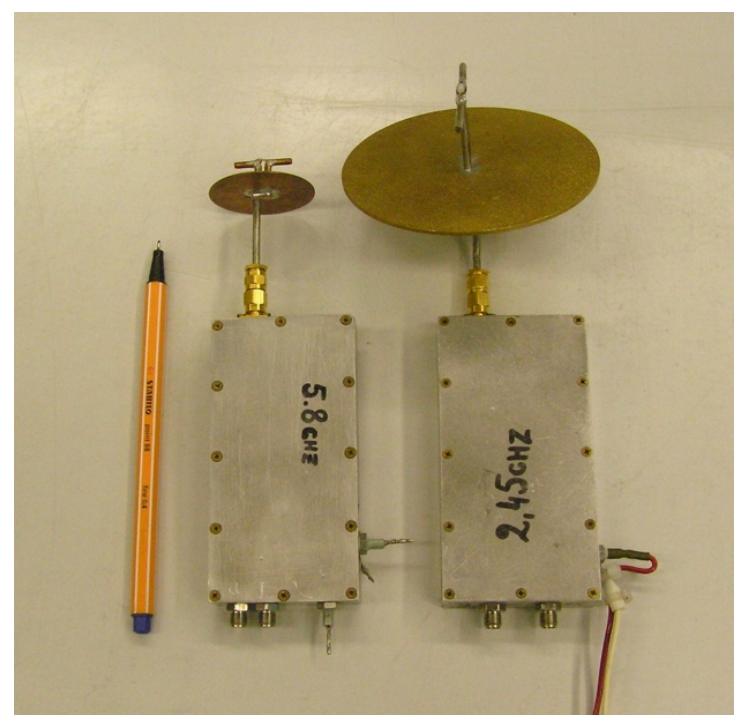

Figure 1. Microwave-sensors developed by Georgian (Caucasus) research institute RPC with fixed frequency $2.45 \mathrm{GHz}$ (right) and $5.8 \mathrm{GHz}$ (left) equipped with halfwave-dipole-antennas. 
time domain and the material density of the asphalt layer (Figure 2). Normally the permittivity is increasing with increasing density in nearly linear manner. Thus according to the Fresnel-equations it is to be expected that the reflection coefficient is increasing with density, too [1] [2]. Indeed, this behavior has been observed in earlier investigations with GPR [6]. However, the GPR-measurements in Figure 2 exhibit the reverse behavior.

To explain this finding a multilayer model developed earlier [7] has been applied. For plane waves and normal incidence it takes into account all transmitted and reflected plane waves, i.e. all interference effects, inside a multilayer system characterized by the complex permittivities (including absorption effects and possible electrical conductivities) and thicknesses of the layers. The amplitudes of the backscattered radar signals in time domain have been calculated exemplary for a variety of thicknesses $d$ and permittivities $\varepsilon_{r}$.

The calculations have been done for a four-layer system consisting of: 1) antenna, 2) lift-off (air gap, $1 \mathrm{~mm}$ ), 3) asphalt and 4) chip board $\left(\varepsilon_{r}=2.5\right)$ resp. asphalt $\left(\varepsilon_{r}=6\right)$ within the frequency band of $1.2 \mathrm{GHz}-3.4 \mathrm{GHz}$. In Figure 3 it can be seen that the magnitude (absolute value) of the reflection coefficient decreases with increasing permittivity of the asphalt layer in a limited permittivity domain even if the frequency bandwidth is relatively big. Hence, due to these interference effects and due to the close correlation of the density with the permittivity, the magnitude can decrease with increasing density. If the bandwidth is further increased the minimum gets weaker and finally disappears. If there is another asphalt layer (instead of the chip board) with a permittivity close to that of the 1st asphalt layer and lying under the 1st layer, the minimum disappear (red curve in Figure 3) since the interference effects are now much less pronounced. So in real roads which are constructed of three or more asphalt layers, no drop but an increase in amplitude is to be expected as has been shown in [6]. In every case after performing a calibration the asphalt density can be determined with a broadband microwave device.

\subsection{Narrowband Measurements}

In the example given above the lift-off was quite small. Other measurements with a bigger lift-off have been

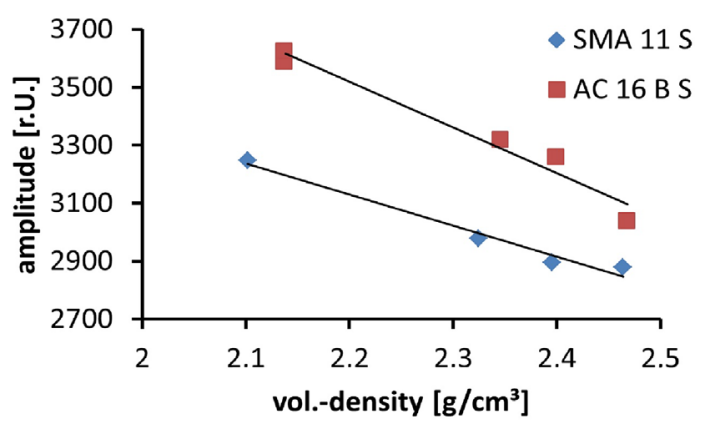

Figure 2. Amplitude (relative units) vs. volume density for asphalt specimens measured with GPR with broadband $2.3 \mathrm{GHz}$ antenna.

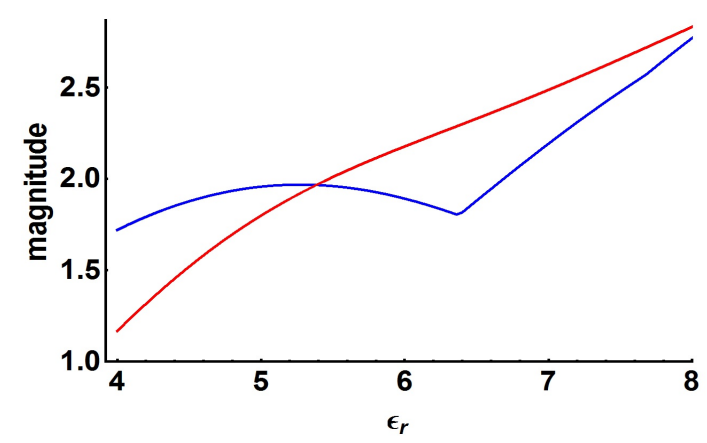

Figure 3. Calculated maximum magnitude of microwave signal in time domain for a variation of permittivity (real part) $\varepsilon_{r}$ for the four-layer system (see text), blue: asphalt layer lying on chip board, red: asphalt layer lying on another asphalt layer. 
performed with the less expensive narrowband sensor incl. a dipole antenna (Figure 1, right). A strong dependence of the measuring amplitude on the lift-off has been observed (see Figure 4 for asphalt type SMA 11 S) since it acts like an additional layer which influences the wave propagation. The curves are oscillating with a periodicity of half wave-length. When the measuring points are plotted vs. density it can be found that some lift-off domains are more favorable than others (Figure 5). The less favorable curves exhibit a maximum or minimum resulting in ambiguities and a reduced sensitivity with respect to permittivity changes of the test object. A clear change in curve slope is unfavorable, too, while the curves measured at a lift-off around $6 \mathrm{~cm}$ exhibit a nearly constant slope and thus a nearly linear behavior (see Figure 6 for both investigated asphalt types).

The influence of the lift-off on the measuring voltage has already been observed earlier [8] [9]. It can be simulated and qualitatively reproduced with the same multi-layer model as mentioned above. In Figure 7 and Figure 8 the layers were: 1) semi-infinite space $\left(\varepsilon_{r}=1\right), 2$ ) layer consisting of varying lift-off $\left.\left(\varepsilon_{r}=1\right), 3\right)$ layer of material with permittivity varying between $\varepsilon_{r}=4$ and 8 and 4) semi-infinite space $\left(\varepsilon_{r}=6\right)$, always for $2.45 \mathrm{GHz}$. With increasing permittivity-corresponding to an increasing material density-the curves in Figure 7 are shifted to the left side in accordance with Figure 4. For Figure 5 and Figure 8 a qualitative compliance between experiment and theory is found with regard to the scattering of the mean magnitude and the shift of its maxima and minima in $\mathrm{x}$-axis.

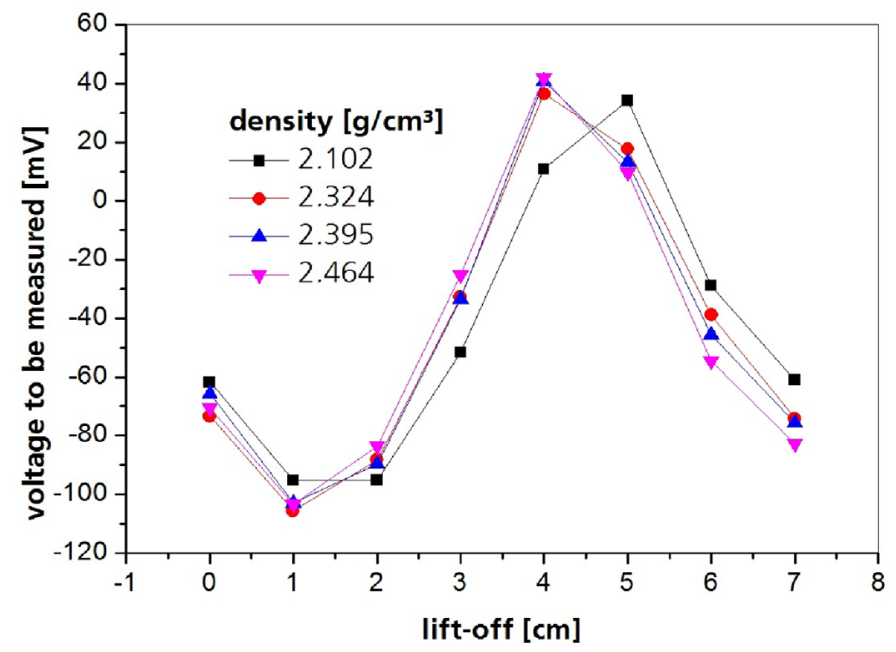

Figure 4. Measured voltage vs. lift-off (SMA 11 S) measured with sensor in Figure 1.

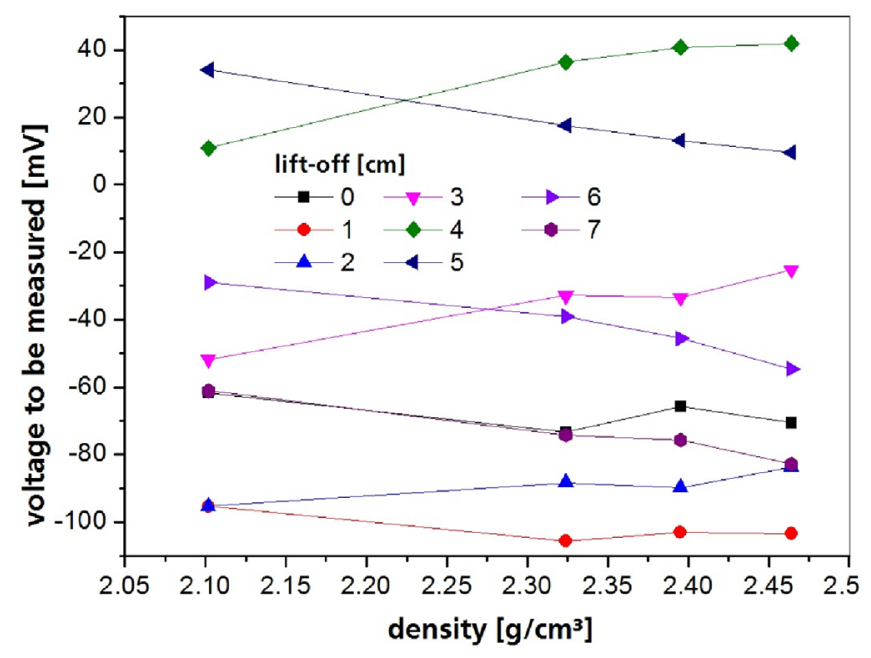

Figure 5. Measured voltage vs. density for different lift-offs (SMA $11 \mathrm{~S}$ ) measured with sensor in Figure 1. 


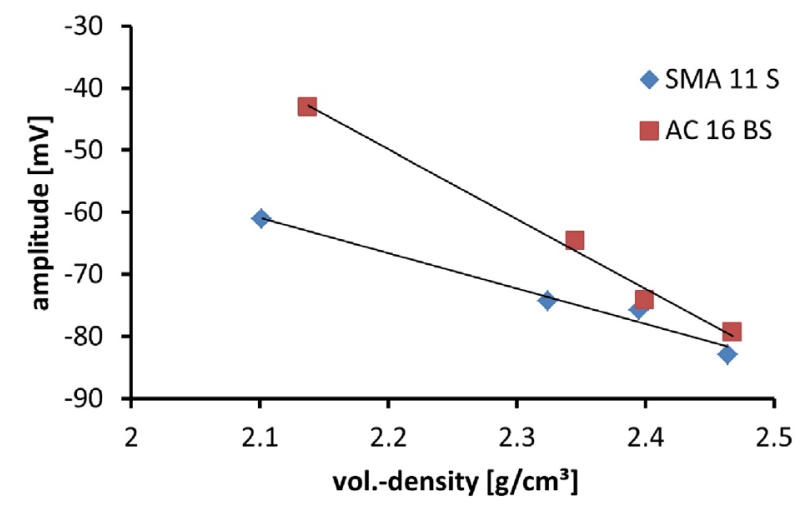

Figure 6. Amplitude vs. volume density for asphalt specimens measured with fixed frequency sensor (2.45 GHz, Figure 1) and dipole antenna (lift-off $7 \mathrm{~cm}$ ).

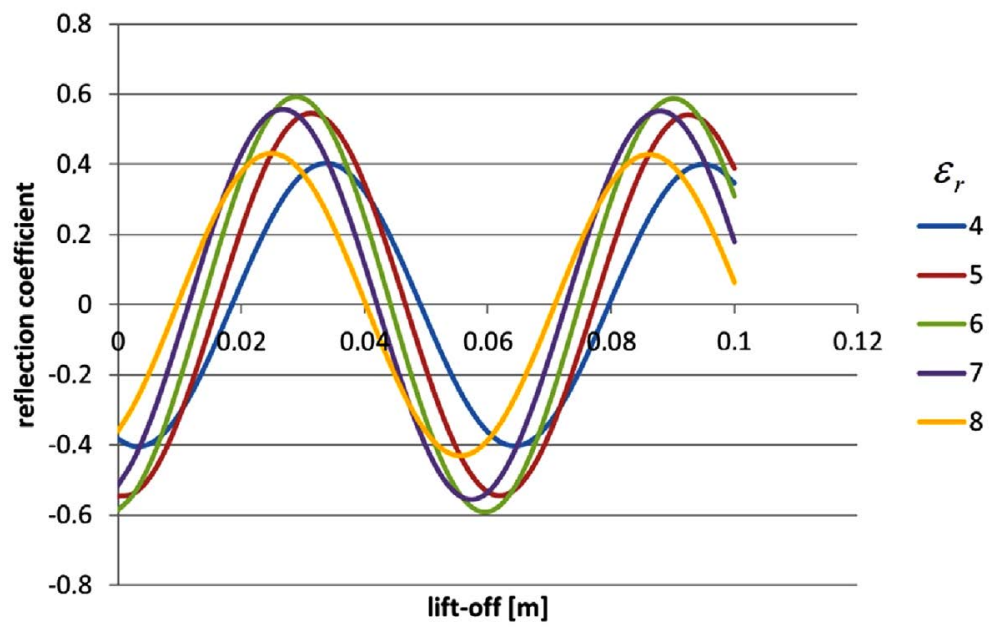

Figure 7. Calculation of the real part of reflection coefficient (narrow band) vs. lift-off for permittivities (real part) varying between $\varepsilon_{r}=4$ and 8 .

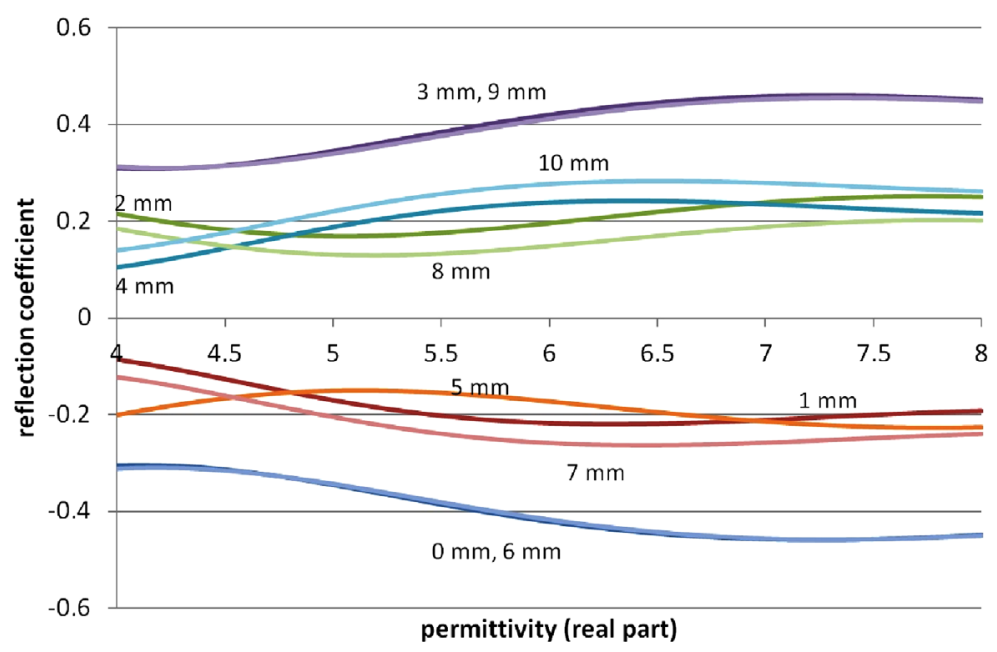

Figure 8. Calculated curves of the real part of the reflection coefficient (narrow band) vs. real part of permittivity of layer 3 with the lift-off as parameter (varying from $0 \mathrm{~mm}$ to $10 \mathrm{~mm}$ ). 
Thus it could be shown that it is possible to measure the density of a solid material with a low-cost microwave sensor provided that a calibration is performed and the lift-off is carefully adjusted.

\section{Conclusion}

The results described above show that there are diverse possibilities to measure the material properties like density nondestructively and contact-free with microwave devices. These encompass both narrowband and broadband devices. Normally a narrowband device is less complex and lower-priced than a broadband one, but it doesn't allow for temporal windowing to eliminate disturbing echoes. These may originate from diverse structural elements of the test object, e.g. from the common steel reinforcements in concrete structures. Particularly for narrowband systems interference effects can occur resulting in partly low sensitivities or ambiguities of the microwave measuring quantity as a function of the material property and geometry. Here the lift-off or air gap between the antenna and the test object can play an important role. It is therefore advisable to maintain a constant lift-off and to optimize it in order to get the best sensitivity with respect to the material and geometrical properties of the test object. If the quantitative knowledge of these properties is wanted a calibration has to be performed. It is necessary to check thoroughly every potential application if a narrowband measurement is sufficient or if a broadband measurement has to be carried out.

\section{References}

[1] Born, M. and Wolf, E. (1986) Principles of Optics. 6th Edition, Pergamon Press, Oxford, New York, Toronto, Sydney, Frankfurt.

[2] Orfanidis, S.J. (2013) Electromagnetic Waves and Antennas. Rutgers University. http://www.ece.rutgers.edu/ orfanidi/ewa/

[3] Kupfer, K. (2010) Aquametry 2010 incl. Feuchtetag 2010, First European Conference on Moisture Measurement in Weimar.

[4] Botsco, R.J., Cribbs, R.W., King, R.J. and McMaster, R.C. (1986) Microwave Methods and Applications in Nondestructive Testing, Section 18, Nondestructive Testing Handbook, 4, Electromagnetic Testing, American Society for Nondestructive Testing.

[5] Zoughi, R. (2000) Microwave Non-Destructive Testing and Evaluation, Non-Destructive Evaluation Series, 4, Kluwer Academic Publishers. http://dx.doi.org/10.1007/978-94-015-1303-6

[6] Saarenketo, T. and Scullion, T. (2000) Journal of Applied Geophysics, 43, 119-138. http://dx.doi.org/10.1016/S0926-9851(99)00052-X

[7] Sklarczyk, C., Ehlen, F. and Netzelmann, U. (1998) Schichten Charakterisieren. Materialprüfung, 40, 149-153.

[8] Baker-Jarvis, J., Janezic, M.D., Domich, P.D., and Geyer, R.G. (1994) Analysis of an Open-Ended Coaxial Probe with Lift-Off for Nondestructive Testing. IEEE Transactions on Instrumentation and Measurement, 43, 711-718. http://dx.doi.org/10.1109/19.328897

[9] Liu, J.M. and Matteson, M.A. (1996) Effects of Lift-Off on Microwave NDE Using an Open-Ended Rectangular Waveguide. Review of Progress in Quantitative Nondestructive Evaluation, 15, 713-718. http://dx.doi.org/10.1007/978-1-4613-0383-1 93 